\title{
Use of Factory-Calibrated Real-time Continuous Glucose Monitoring Improves Time in Target and HbA1c in a Multiethnic Cohort of Adolescents and Young Adults With Type 1 Diabetes \\ DOI:
}

10.2337/dc20-0736

\section{Document Version}

Accepted author manuscript

Link to publication record in Manchester Research Explorer

\section{Citation for published version (APA):}

Thabit, H., Prabhu, J. N., Mubita, W., Fullwood, C., Azmi, S., Urwin, A., Doughty, I., \& Leelarathna, L. (2020). Use of Factory-Calibrated Real-time Continuous Glucose Monitoring Improves Time in Target and $\mathrm{HbA} 1 \mathrm{c}$ in a Multiethnic Cohort of Adolescents and Young Adults With Type 1 Diabetes: The MILLENNIALS Study. Diabetes Care, 43(10), 2537-2543. https://doi.org/10.2337/dc20-0736

\section{Published in:}

Diabetes Care

\section{Citing this paper}

Please note that where the full-text provided on Manchester Research Explorer is the Author Accepted Manuscript or Proof version this may differ from the final Published version. If citing, it is advised that you check and use the publisher's definitive version.

\section{General rights}

Copyright and moral rights for the publications made accessible in the Research Explorer are retained by the authors and/or other copyright owners and it is a condition of accessing publications that users recognise and abide by the legal requirements associated with these rights.

\section{Takedown policy}

If you believe that this document breaches copyright please refer to the University of Manchester's Takedown Procedures [http://man.ac.uk/04Y6Bo] or contact uml.scholarlycommunications@manchester.ac.uk providing relevant details, so we can investigate your claim.

\section{OPEN ACCESS}


Use of Factory-Calibrated Real-time Continuous Glucose Monitoring Improves Time in Target and $\mathrm{HbA}_{1 \mathrm{c}}$ in a Multiethnic Cohort of Adolescents and Young Adults With Type 1 Diabetes: The MILLENNIAL Study

Diabetes Care 2020;43:1-7 | https://doi.org/10.2337/dc20-0736
Hood Thabit, ${ }^{1,2}$ Joshi Navis Prabhu, ${ }^{1}$ Womba Mubita, ${ }^{1}$ Catherine Fullwood, ${ }^{3}$ Shazli Azmi, ${ }^{1}$ Andrea Urwin, ${ }^{1}$ lan Doughty, ${ }^{4}$ and Lalantha Leelarathna ${ }^{1,2}$

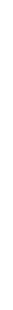

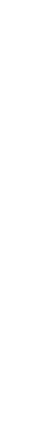

\begin{abstract}
OBJECTIVE
International type 1 diabetes registries have shown that $\mathrm{HbA}_{1 \mathrm{c}}$ levels are highest in young people with type 1 diabetes; however, improving their glycemic control remains a challenge. We propose that use of the factory-calibrated Dexcom G6 CGM system would improve glycemic control in this cohort.
\end{abstract}

\section{RESEARCH DESIGN AND METHODS}

We conducted a randomized crossover trial in young people with type 1 diabetes (16-24 years old) comparing the Dexcom G6 CGM system and self-monitoring of blood glucose (SMBG). Participants were assigned to the interventions in random order during two 8-week study periods. During SMBG, blinded continuous glucose monitoring (CGM) was worn by each participant for 10 days at the start, week 4 , and week 7 of the control period. $\mathrm{HbA}_{1 \mathrm{c}}$ measurements were drawn after enrollment and before and after each treatment period. The primary outcome was time in range 70-180 $\mathrm{mg} / \mathrm{dL}$.

\section{RESULTS}

Time in range was significantly higher during CGM compared with control (35.7 \pm $13.5 \%$ vs. $24.6 \pm 9.3 \%$; mean difference $11.1 \%$ [95\% Cl 7.0-15.2]; $P<0.001$ ). CGM use reduced mean sensor glucose $(219.7 \pm 37.6 \mathrm{mg} / \mathrm{dL}$ vs. $251.9 \pm 36.3 \mathrm{mg} / \mathrm{dL}$; mean difference $-32.2 \mathrm{mg} / \mathrm{dL}[95 \% \mathrm{Cl}-44.5$ to -20.0$] ; P<0.001$ ) and time above range (61.7 $\pm 15.1 \%$ vs. $73.6 \pm 10.4 \%$; mean difference $11.9 \%$ [95\% $\mathrm{Cl}-16.4$ to -7.4$] ; P<$ 0.001). $\mathrm{HbA}_{1 \mathrm{c}}$ level was reduced by $0.76 \%(95 \% \mathrm{Cl}-1.1$ to -0.4$)(-8.5 \mathrm{mmol} / \mathrm{mol}$ [95\% Cl -12.4 to -4.6$] ; P<0.001)$. Times spent below range $(<70 \mathrm{mg} / \mathrm{dL}$ and $<54 \mathrm{mg} / \mathrm{dL}$ ) were low and comparable during both study periods. Sensor wear was $84 \%$ during the CGM period.

\section{CONCLUSIONS}

CGM use in young people with type 1 diabetes improves time in target and $\mathrm{HbA}_{1 \mathrm{c}}$ levels compared with SMBG.
${ }^{1}$ Manchester Diabetes Centre, Manchester Royal Infirmary, Manchester, U.K.

${ }^{2}$ Division of Diabetes, Endocrinology and Gastroenterology, Faculty of Biology, Medicine and Health, University of Manchester, Manchester, U.K.

${ }^{3}$ Research and Innovation, Manchester Royal Infirmary, Manchester, U.K.

${ }^{4}$ Royal Manchester Children's Hospital, Manchester Royal Infirmary, Manchester, U.K.

Corresponding author: Hood Thabit, hood.thabit@ mft.nhs.uk

Received 4 April 2020 and accepted 29 June 2020

Clinical trial reg. no. NCT03445377, clinicaltrials gov

This article contains supplementary material online at https://doi.org/10.2337/figshare .12616208 .

(C) 2020 by the American Diabetes Association. Readers may use this article as long as the work is properly cited, the use is educational and not for profit, and the work is not altered. More information is available at https://www.diabetesjournals .org/content/license. 
The goal of type 1 diabetes management is the attainment of optimal glycemic control to avoid acute and longer-term complications (1). Recent international type 1 diabetes registries have shown that $\mathrm{HbA}_{1 \mathrm{c}}$ levels are highest in adolescents and young adults $(2,3)$, with mean $\mathrm{HbA}_{1 \mathrm{c}}$ levels in this cohort increasing from 2010-2012 to 2016-2018. Glycemic control earlier in life may have direct consequence on future diabetes-related comorbidities (4). This is compounded by the increasing duration of diabetes as this cohort becomes older. Emerging evidence also reports that during this challenging period of their lives, young people living with type 1 diabetes can experience significant psychosocial burden, which adversely impacts their psychological adjustment and diabetes management $(5,6)$.

Real-time continuous glucose monitoring (RT-CGM) is associated with lower $\mathrm{HbA}_{1 \mathrm{c}}$ levels, with benefits linked to user adherence of the technology (7-9). The improvement in glycemic control, however, appears to be attenuated in youths and adolescents compared with other age groups. The JDRF CGM study reported that only $30 \%$ of adolescents and young adults (15-24 years) were using CGM for $\geq 6$ days/week, compared with $83 \%$ of adults ( $>25$ years old) (8). Recent reports also showed that actual rates of CGM use in clinical practice are lowest in adolescents and young adults, irrespective of reimbursement (9). There is therefore an unmet need to find innovative strategies to address the adoption of CGM and evaluate its efficacy in this vulnerable group. The advent of the Dexcom G6 factory-calibrated CGM system, which is linked to a smartphone application (app), appears promising and may provide a novel approach to the above need (10).

In this study, we present the results of an 8-week randomized controlled trial in adolescents and young adults aged 16-25 years with suboptimally controlled type 1 diabetes. We hypothesized that use of a factory-calibrated CGM system with smartphone app in this cohort would improve glycemic control compared with selfmonitoring of blood glucose (SMBG).

\section{RESEARCH DESIGN AND METHODS}

\section{Study Design and Participants}

The study had an open-label, randomized controlled crossover study design.

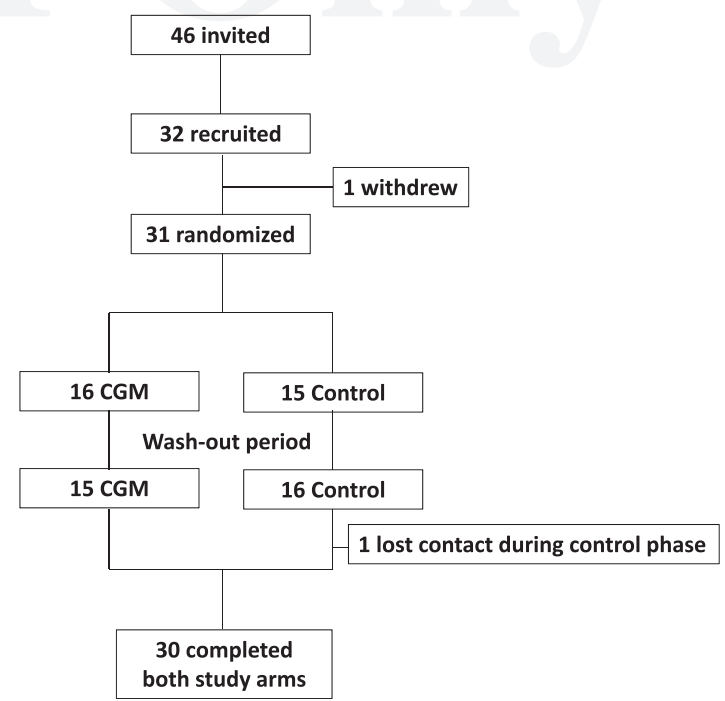

Figure 1-Flow of participants.

All participants had type 1 diabetes as defined by the World Health Organization for at least 1 year and were either on multiple daily injections (MDI) or insulin pump therapy. We identified and recruited adolescents and young adult participants between 16 and 24 years of age attending Royal Manchester Children Hospital, Manchester Royal Infirmary Hospital (Manchester, U.K.) and Trafford General Hospital (Manchester, U.K.). Other inclusion and exclusion criteria are provided in the Supplementary Appendix.

Recruited participants underwent a 2-week run-in period with a blinded
CGM sensor to assess tolerance to wearing the sensor. All participants who successfully completed the run-in period were then randomly assigned to 8 weeks of CGM using the Dexcom G6 system during the intervention period and SMBG during the control period. Randomization (1:1 block) was performed by a centralized web-based program.

Participants installed the Dexcom G6 Mobile CGM app in their own personal smartphone and were given a Dexcom G6 receiver as backup. Prior to CGM use, participants were trained and educated on CGM application and decision-making
Table 1-Characteristics of the study participants at baseline Characteristics

\begin{tabular}{|c|c|}
\hline \multicolumn{2}{|l|}{ Sex, $N(\%)$} \\
\hline Female & $18(58.1)$ \\
\hline Male & 13 (41.9) \\
\hline Age (years) & $21.2 \pm 2.3$ \\
\hline Age range $16-20$ years, $N(\%)$ & $14(45)$ \\
\hline Age range $21-24$ years, $N(\%)$ & $17(55)$ \\
\hline BMI $\left(\mathrm{kg} / \mathrm{m}^{2}\right)$ & $25.5 \pm 4.6$ \\
\hline Duration of diabetes (years), median (IQR) & $12.9(9.0-16.4)$ \\
\hline \multicolumn{2}{|l|}{ Insulin delivery method, $N(\%)$} \\
\hline MDI & $14(45.2)$ \\
\hline Insulin pump therapy & $17(54.8)$ \\
\hline $\mathrm{HbA}_{1 \mathrm{c}}$ at screening, $\%(\mathrm{mmol} / \mathrm{mol})$ & $9.3 \pm 1.3(78.4 \pm 14.4)$ \\
\hline \multicolumn{2}{|l|}{ Ethnicity, $N(\%)$} \\
\hline White & $20(64.5)$ \\
\hline Black British/African/Caribbean & $6(19.4)$ \\
\hline Asian British/Asian & $2(6.5)$ \\
\hline Mixed/multiple ethnic groups & $3(9.7)$ \\
\hline Index of Multiple Deprivation rank, median (IQR) & $7,261(2,354-13,804)$ \\
\hline Index of Multiple Deprivation decile, median (IQR) & $3.0(1.0-5.0)$ \\
\hline
\end{tabular}

Data are mean \pm SD unless otherwise indicated. 
Table 2-Glycemic outcomes for the whole study period and sensor wear

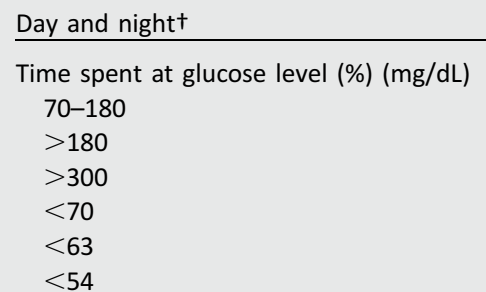

\section{$\mathrm{HbA}_{1 \mathrm{c}}$ change (\%)}

$\mathrm{HbA}_{1 \mathrm{c}}$ change $(\mathrm{mmol} / \mathrm{mol})$

Mean glucose $(\mathrm{mg} / \mathrm{dL})$

SD of glucose $(\mathrm{mg} / \mathrm{dL})$ CGM $(n=30)$ Control $(n=30)$ Paired mean difference $(95 \% \mathrm{Cl})^{*}$ $P$ value

$\mathrm{CV}$ of glucose (\%)

$35.7 \pm 13.5$
$61.7 \pm 15.1$
$21.7 \pm 14.4$
$1.45(0.40-4.08)$
$0.65(0.18-2.32)$
$0.28(0.05-1.16)$
$-0.53 \pm 0.74$
$-5.9 \pm 8.0$
$219.7 \pm 37.6$
$85.3 \pm 14.1$
$39.3 \pm 5.6$

$11.1(7.0-15.2)$
$-11.9(-16.4,-7.4)$
$-11.7(-16.8,-6.6)$

$-0.76(-1.1,-0.4)$
$-8.5(-12.4,-4.6)$
$-32.2(-44.5,-20.0)$
$-4.6(-9.0,-0.3)$
$3.0(1.0-4.9)$

$<0.001$
$<0.001$
$<0.001$
0.055
0.111
0.102
$<0.001$
$<0.001$
$<0.001$
0.037
0.006

Data are mean \pm SD or median (IQR). CV, coefficient of variation. *Normally distributed data are presented as mean differences of values (CGM minus control phase). A positive value indicates that the measurement was higher during the CGM period than it was during the control period.

using the manufacturer's printed training guide. Competency on each participant's decision-making capability using CGM data was assessed at the end of the CGM training period. Participants performed fingerstick capillary glucose measurements as per usual clinical practice during the 8-week SMBG control period. During the control period, a blinded CGM (glucose values not displayed to participant) was worn by each participant for 10 days at the start, week 4, and week 7 of the control period. The two treatment periods were separated by a 3- to 4-week washout, during which they continued with their standard method of capillary glucose monitoring.

\section{Outcomes}

The primary end point in the study was the proportion of time that the sensor glucose level as measured by the CGM device was in the target range of 3.9$10 \mathrm{mmol} / \mathrm{L}$ during the 8-week study period. Secondary end points were the glycated hemoglobin level, mean sensor glucose levels, and the time spent below and above the relevant glucose ranges during day and night (daytime [0800$2300 \mathrm{~h}$ ] and overnight [2300-0800 h]).

\section{Data Collection}

Participants had identical planned contact with the study team during the two treatment periods. CGM data pertaining to primary and secondary end points were collected and exported for analysis using the manufacturer's designated web-based Dexcom CLARITY software. Blood samples for $\mathrm{HbA}_{1 \mathrm{c}}$ measurements were drawn after enrollment and before and after each treatment period. $\mathrm{HbA}_{1 \mathrm{c}}$ analysis was performed at a central laboratory. Information pertaining to usability and psychosocial impact of using CGM in this age group was collected using validated questionnaires, which were given to participants at recruitment and end of the second treatment period. Other end points collected were the number of self-measurements of blood glucose and rate of severe hypoglycemia, defined as unconsciousness from hypoglycemia or requiring assistance from another person. Participants were assigned an English Index of Deprivation rank (https://www .gov.uk/government/statistics/englishindices-of-deprivation-2019) based upon their postcode. These were then grouped by deprivation decile defined by their position in the ranks from the 32,844 small areas in England subdivided into 10 equal groups, with 1 being the most socioeconomically deprived and 10 being the least deprived.

\section{Sample Size}

The power calculation was based on improvements in time in target. Assuming an SD of $18 \%$ and average improvement of time in target of $10 \%, 30$ subjects are required at the desired $80 \%$ power and an $\alpha$ level of 0.05 (two-tailed). Up to 40 subjects were planned to be recruited, aiming for 30 completed subjects to allow for dropouts. Subjects who dropped out of the study during run-in period and within the first 2 weeks of the first intervention were allowed to be replaced.

\section{Ethics and Support Sources}

The study protocol was approved by an independent research ethics committee (REC reference: $18 / \mathrm{NW} / 0183$ ). All participants provided written informed consent. Dexcom, Inc. provided funding and supplied the CGM devices and sensors used in the study. Dexcom, Inc. read the manuscript before submission. No sponsor had any role in the study design, data collection, data analysis, data interpretation, or writing of the report. The corresponding author had full access to all study data and had final responsibility for the decision to submit the publication.

\section{Statistical Analysis}

Statistical analyses were performed on an intention-to-treat basis. Any period effect resulting from the intervention and control periods was investigated using univariate ANOVA adjusted for period and subject. As no period effect was found, paired-sample $t$ tests and Wilcoxon signed-rank tests were performed for normal and nonnormal variables, respectively. Values were presented as mean \pm SD or median (interquartile range [IQR]). The hypothesis testing was ordered first to consider the primary end point at the 0.05 level and then to move to testing the secondary end points individually at the 0.05 level without any control for multiplicity. We did the analyses with SPSS (version 25; IBM Software, Hampshire, U.K.). All $P$ values are two-sided.

\section{RESULTS}

\section{Participants}

Forty-six potential participants were invited to the study. Fourteen did not reply or were unable to participate due to other commitments. Thirty-two participants were recruited from September 2018 to July 2019. Figure 1 shows the 


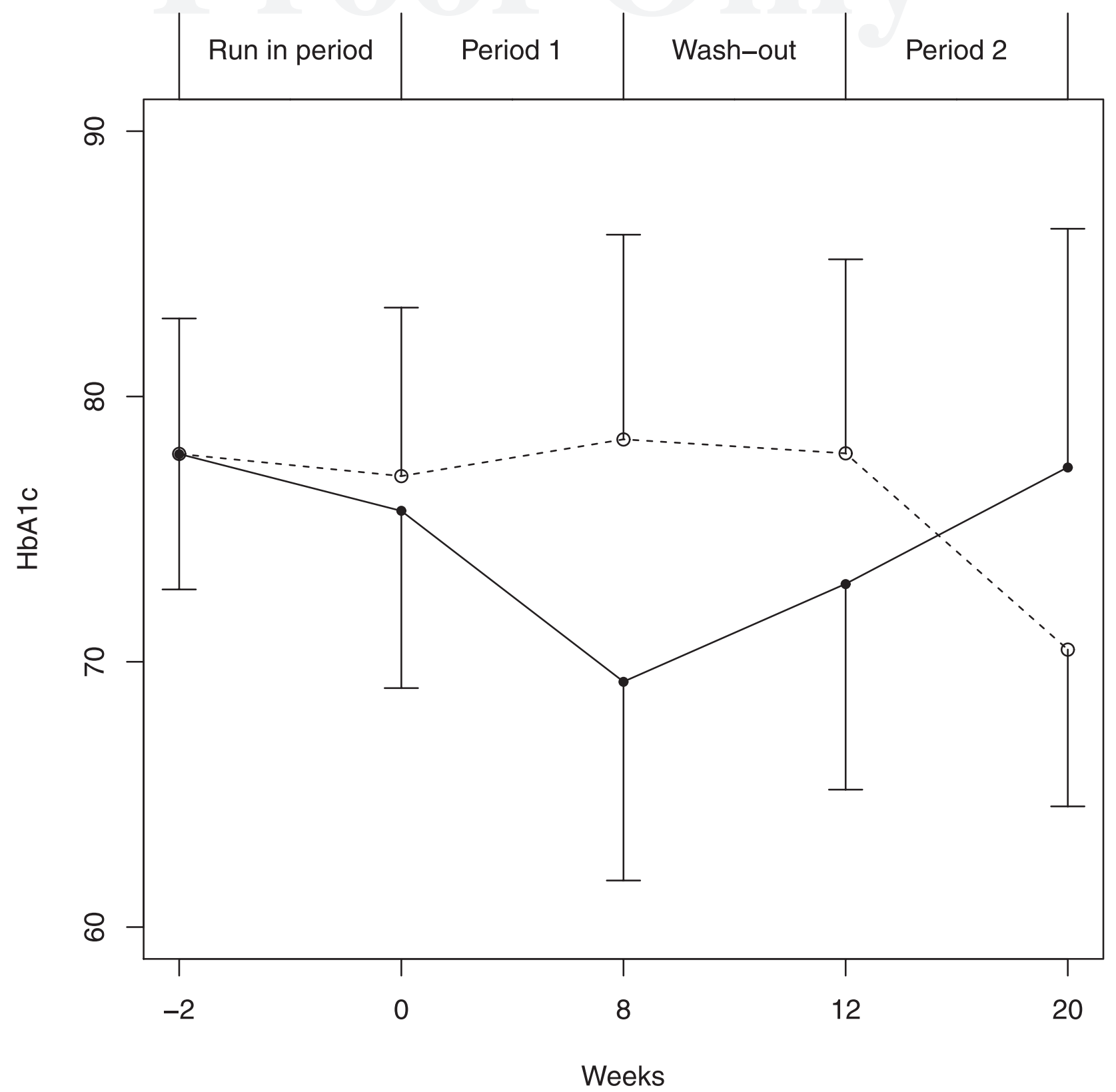

Figure 2- $\mathrm{HbA}_{1 \mathrm{c}}$ changes during study period. Solid line indicates CGM first, followed by control intervention; dashed line indicates control first, followed by CGM intervention.

flow of participants through the study. One participant withdrew during the run-in period before being randomized due to work commitments. Another participant was uncontactable during the control period; however, the participant returned for the final visit, and an $\mathrm{HbA}_{1 \mathrm{c}}$ sample was taken.

Table 1 summarizes baseline characteristics of randomized participants. $\mathrm{HbA}_{1 \mathrm{c}}$ at screening was $9.3 \pm 1.3 \%$ (78.4 \pm $14.4 \mathrm{mmol} / \mathrm{mol}$ ). The numbers of participants on MDI or insulin pump therapy were comparable. Sixty-five percent selfidentified as white British, and 58\% lived in areas of relatively high deprivation (the most deprived $30 \%$ of small areas in England).

\section{Study Primary and Secondary End Points \\ Twenty-Four-Hour Period}

Overall 24-h glucose control over 8 weeks during the CGM and control periods is shown in Table 2. The primary end point of the study, the proportion of time during the 24-h period when sensor glucose was in target range from 70 to $180 \mathrm{mg} / \mathrm{dL}$, was significantly increased by 11.1 percentage points (95\% Cl 7.0-15.2\%; $P<0.001$ ) during the CGM period. CGM use reduced mean sensor glucose by $32.2 \mathrm{mg} / \mathrm{dL}$ (95\% $\mathrm{Cl}-44.5$ to $-20.0 \mathrm{mg} / \mathrm{dL} ; P<0.001)$ and time spent above target by 11.9 percentage points $(95 \% \mathrm{Cl}-16.4$ to $-7.4 \%$; $P<$ $0.001)$. The proportion of time sensor glucose level in the hypoglycemia range was relatively low and comparable during both study periods.

\section{$H b A_{1 c}$ Change}

$\mathrm{HbA}_{1 \mathrm{c}}$ level reduced by $0.53 \pm 0.74 \%$ ( $5.9 \pm 8.0 \mathrm{mmol} / \mathrm{mol}$ ) during the CGM period and increased by $0.24 \pm 0.69 \%$ (2.6 \pm $7.5 \mathrm{mmol} / \mathrm{L}$ ) during the control period 
Table 3-Glycemic outcomes during nighttime and daytime periods

\begin{tabular}{|c|c|c|c|c|}
\hline & CGM & Control & Paired mean difference $(95 \% \mathrm{Cl})^{*}$ & $P$ value \\
\hline \multicolumn{5}{|c|}{ Nighttime only $(2300-0800 \mathrm{~h}) \ddagger$} \\
\hline Mean glucose (mg/dL) & $218.7 \pm 37.3$ & $251.7 \pm 41.8$ & $-33.0(-46.4,-19.6)$ & $<0.001$ \\
\hline \multicolumn{5}{|c|}{ Time spent at glucose level (\%) (mg/dL) } \\
\hline $70-180$ & $34.9 \pm 13.8$ & $25.3 \pm 11.9$ & $9.7(4.7-14.7)$ & $<0.001$ \\
\hline 70-144 & $22.7 \pm 10.4$ & $13.6 \pm 7.4$ & $9.1(5.7-12.5)$ & $<0.001$ \\
\hline$>180$ & $62.4 \pm 15.5$ & $73.0 \pm 12.8$ & $-10.5(-15.9,-5.1)$ & $<0.001$ \\
\hline$>144$ & $74.6 \pm 12.4$ & $84.6 \pm 9.1$ & $-10.0(-13.9,-6.1)$ & $<0.001$ \\
\hline$<70$ & $1.06(0.51-4.03)$ & $0.73(0.05-3.56)$ & & 0.055 \\
\hline$<50$ & $0.10(0.00-0.63)$ & $0.00(0.00-0.44)$ & & 0.117 \\
\hline \multicolumn{5}{|c|}{ Daytime only $\ddagger(0800-2300$ h) } \\
\hline Mean glucose $(\mathrm{mg} / \mathrm{dL})$ & $218.3 \pm 39.5$ & $249.4 \pm 40.8$ & $-31.1(-42.4,-19.7)$ & $<0.001$ \\
\hline \multicolumn{5}{|c|}{ Time spent at glucose level (\%) (mg/dL) } \\
\hline $70-180$ & $36.8 \pm 13.9$ & $25.5 \pm 10.9$ & $11.3(7.2-15.4)$ & $<0.001$ \\
\hline$>180$ & $60.3 \pm 15.5$ & $72.3 \pm 12.5$ & $-12.0(-16.7,-7.3)$ & $<0.001$ \\
\hline$<70$ & $2.03(0.53-4.05)$ & $0.94(0.22-3.50)$ & & 0.179 \\
\hline$<50$ & $0.27(0.00-0.99)$ & $0.02(0.00-0.77)$ & & 0.408 \\
\hline
\end{tabular}

Data are mean \pm SD or median (IQR). *Normally distributed data are presented as mean differences of values (CGM minus control phase). A positive value indicates that the measurement was higher during the CGM period than it was during the control period.

(mean difference CGM vs. control; $-0.76 \%$ [95\% Cl -1.1 to -0.4$][-8.5 \mathrm{mmol} / \mathrm{mol}$ (95\% Cl -12.4 to -4.6$) ; P<0.001])$. $\mathrm{HbA}_{1 \mathrm{c}}$ was lower in participants on CGM during the first and second treatment periods (Fig. 2).

\section{Nighttime and Daytime Period}

Overnight and daytime glycemic end points are shown in Table 3 . The proportion of time that the glucose level was within the overnight target range was significantly increased by 9.1 percentage points (95\% Cl 5.7-12.5; $P<0.001$ ) with CGM, and this was associated with reduced time spent above the overnight target range and mean sensor glucose level. The proportions of time spent in hypoglycemia were relatively low and comparable during both CGM and control periods.

Compared with the control period, CGM significantly increased the proportion of time that the glucose level was within daytime target range by 11.3 percentage points $(95 \% \mathrm{Cl}$ 7.2-15.4; $P<$ 0.001 ). Similarly, time spent above daytime target range and mean sensor glucose level were significantly reduced by CGM. The proportions of time spent in hypoglycemia during daytime were comparable between the CGM and control periods.

Treatment Adherence and User Feedback Overall median time and IQR of CGM use, estimated by the proportion of CGM data downloaded in relation to follow-up time, was $84.0 \%$ (62.6-94.0). Compared with the control arm, participants using RT-CGM had significantly higher total mean score on the Glucose Monitoring Satisfaction
Survey ( $4.0 \pm 0.6$ vs. $3.5 \pm 0.6 ; P=0.011)$, indicating greater satisfaction with RT-CGM. Participants on RT-CGM reported greater openness ("Openness" item mean score $3.6 \pm 0.9$ vs. $2.9 \pm 0.9 ; P=0.003$ ) and reduced burden ("Behavioral Burden" item mean score $1.8 \pm 0.7$ vs. $2.4 \pm 0.9$; $P=0.007)$. The mean score for the Glucose Monitoring Satisfaction Survey items "Emotional Burden" and "Trust" was comparable for the RT-CGM and control arms. There was no significant difference in total Diabetes Distress Scale score between both groups.

\section{SMBG}

Patients performed an average of $2.8 \pm$ 1.4 self-measurements of blood glucose during CGM therapy and $3.7 \pm 2.3$ during conventional therapy.

\section{Adverse Events}

Details of all of the adverse events are provided in Table 4. No episodes of severe hypoglycemia or diabetes ketoacidosis occurred in either study period. One episode of diabetes ketoacidosis occurred during the washout period due to an intercurrent illness.

\section{CONCLUSIONS}

Our findings show that in this randomized controlled trial, use of CGM in a multiethnic cohort of adolescents and young adults with type 1 diabetes led to a significant increase in the proportion of time that the glucose level was in the target range compared with SMBG. This was accompanied by significant reduction in $\mathrm{HbA}_{1 \mathrm{c}}$ level, mean glucose, and time spent in hyperglycemia. The benefits in glycemic outcome over the whole 24-h period were also seen separately during overnight and daytime periods.

Data from international registries have consistently reported higher $\mathrm{HbA}_{1 \mathrm{c}}$ levels among adolescents and young adults with type 1 diabetes compared with preadolescents and older adults $(2,3,11)$. Challenges faced by this age group in achieving their glycemic targets include lower engagement levels of diabetes

Table 4-Adverse events

Events RT-CGM period Control period Washout period

Gastroenteritis

Respiratory tract infection

Urinary tract infection

Ketonemia related to missed

basal insulin injection

Fractured foot

Diabetes ketoacidosis due to

intercurrent illness

$\begin{array}{lll}1 & 2 & 0 \\ 0 & 2 & 0 \\ 0 & 1 & 0\end{array}$

100

100

0

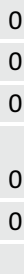

0 
self-management and the psychological burden of living with the condition during this developmental phase of their lives $(12,13)$. Although previous randomized studies have shown clinically meaningful $\mathrm{HbA}_{1 \mathrm{c}}$ reduction during CGM use compared with SMBG $(7,14,15)$, the majority of these studies were performed in adults, with relatively few randomized studies in adolescents and young adults. Observational nonrandomized studies showed that CGM use in adolescents with type 1 diabetes facilitated $\mathrm{HbA}_{1 \mathrm{c}}$ improvement $(2,16)$, while a meta-analysis showed no difference in $\mathrm{HbA}_{1 \mathrm{c}}$ levels between CGM and SMBG in this cohort (17). These results, however, are limited by the inherent risk of bias in the former and the heterogeneity between studies in the latter.

To our knowledge, this is the first randomized clinical study of a factorycalibrated CGM system in this age group. The reduced daily burden of using factory-calibrated CGM, which is clinically approved for nonadjunctive use as part of diabetes management (18), may have been a contributory factor associated with the greater satisfaction and openness reported in this study, as well as reduced daily hassle associated with the device. The smartphone app use may have also facilitated the CGM use adherence observed in our study (median use $84 \%$ compared with $30 \%$ in the JDRF CGM study in adolescence) by reducing the need to carry additional devices. Device burden is known to be associated with discontinuation of CGM use in this cohort (19). We therefore speculate that these factors may have attributed to the relatively higher percentage of CGM sensor use in our study compared with others. In JDRF CGM study, although higher sensor use adherence was associated with greater $\mathrm{HbA}_{1 \mathrm{c}}$ reduction (20), sensor use and $\mathrm{HbA}_{1 \mathrm{c}}$ reduction were significantly lower among adolescents compared with adults (8). Certain caveats, however, need to be considered when comparing our findings with older CGM studies, as reported outcomes are also likely influenced by recent improvements in CGM system technology, such as sensor accuracy and usability.

The current work extends findings from adult studies using a similar Dexcom G6 CGM system, which showed improvement in glycemic control compared with SMBG among $\mathrm{MDI}(7,14)$ and pump users $(15)$. Participants in our study were provided with individualized training and approved educational materials to help optimize their use of the CGM system. Long-term adherence to health care professional and educator advice, however, in this specific age group is known to be lower compared with the older group and may have influenced the moderate improvement of time in target observed in our study. Although the time in target achieved by CGM in our study was relatively low compared with that reported by others, the reduction in $\mathrm{HbA}_{1 \mathrm{c}}$ achieved is nevertheless clinically meaningful in this young population in the reduction of the risk of future complications. There was a trend of numerically increased hypoglycemia rates during CGM use, which did not reach statistical significance. However, in the context of the relatively low hypoglycemia rates during the control period at the expense of hyperglycemia (time spent $<70 \mathrm{mg} / \mathrm{dL}$ of $9 \mathrm{~min} /$ day during control period and $20 \mathrm{~min} /$ day during CGM period) is not unexpected and comparable to the GOLD study in adults with type 1 diabetes (mean \pm SD $2.79 \pm 2.97 \%$ ). We also noted slight increase in glucose variability in our study. This is likely explained by participants correcting high glucose levels in the CGM period compared with persistent underrecognized and therefore uncorrected high glucose levels in the control period.

The strengths of our study are the randomized crossover design; confounding study period effect was not detected for the primary end point. Compared with other published type 1 diabetes and technology studies, ours included a higher proportion of participants from diverse ethnic backgrounds, who tend to be underrepresented in type 1 diabetes research and practice (21). Over half of our participants also live in areas constituting the $30 \%$ most deprived areas of England. Our study thus allows for greater generalizability, especially among inner-city inhabitants with type 1 diabetes. We included both MDI and pump users, in keeping with current use of CGM in both treatment modalities. High adherence to glucose sensor wear during the CGM period was observed, likely due to reduced device burden. The study was limited by the relatively short duration, restricting longer-term assessment. The sample size of the study limits the statistical power to perform subgroup analysis (i.e., glycemic outcomes among $\mathrm{MDI}$ /pump users and ethnicity). The amount of sensor data available for analysis was not equal between the CGM and control periods; however, the $\mathrm{HbA}_{1 \mathrm{c}}$ level improvement supports the glycemic benefit of CGM use in this population.

In conclusion, we found that use of a factory-calibrated Dexcom G6 CGM system in adolescents and young adults with type 1 diabetes over an 8-week period led to an increase in proportion of time spent in target range and reduced time spent in hyperglycemia, accompanied by meaningful $\mathrm{HbA}_{1 \mathrm{c}}$ reduction. This was associated with greater satisfaction in glucose monitoring, further extending its benefit within this population.

Acknowledgments. The authors thank the study volunteers for participation in the studies and acknowledge support by the staff at Manchester Diabetes Centre and Royal Manchester Children's Hospital (Manchester, U.K.).

Funding. Dexcom, Inc. provided funding and supplied the CGM devices and sensors used in the study.

Dexcom, Inc. read the manuscript before submission. No sponsor had any role in the study design, data collection, data analysis, data interpretation, or writing of the report.

Duality of Interest. H.T. has received research Q:16 support from Dexcom, Inc. S.A. received honoraria for educational speaker meetings from Novo Nordisk, AstraZeneca, Boehringer Ingelheim/Eli Q:10 Lilly and Company, Napp Pharmaceuticals, and Sanofi. L.L. has received speaker honoraria from Animas, Abbott, Insulet, Medtronic, Novo Nordisk, Roche, and Sanofi; served on advisory panels for Animas, Abbott, Novo Nordisk, Dexcom, Inc., Medtronic, Sanofi, and Roche; and received research support from Novo Nordisk and Dexcom, Inc. No other potential conflicts of interest relevant to this article were reported.

Author Contributions. H.T. and L.L. designed the study. H.T., J.N.P., W.M., S.A., I.D., and L.L. screened and enrolled participants and arranged informed consent from the participants. H.T., J.N.P., W.M., A.U., and L.L. provided patient care and took samples. H.T. managed randomization. H.T., C.F., and L.L. did or supported data analyses, including the statistical analyses. H.T., C.F., and L.L. interpreted the results. H.T. wrote the manuscript. All authors critically reviewed the report. No writing assistance was provided. H.T. and L.L. are the guarantors of this work and, as such, had full access to all of the data in the study and take responsibility for the integrity of the data and the accuracy of the data analysis.

Prior Presentation. Parts of this work were presented in poster form at the 80th Scientific Sessions of the American Diabetes Association, 12-16 June 2020.

\section{References}

1. DiMeglio LA, Evans-Molina C, Oram RA. Type 1 diabetes. Lancet 2018;391:2449-2462

2. Foster NC, Beck RW, Miller KM, et al. State of type 1 diabetes management and outcomes from 
the T1D Exchange in 2016-2018. Diabetes Technol Ther 2019;21:66-72

3. Schwandt A, Hermann JM, Rosenbauer J, et al.; DPV Initiative. Longitudinal trajectories of metabolic control from childhood to young adulthood in type 1 diabetes from a large German/Austrian registry: a group-based modeling approach. Diabetes Care 2017;40:309-316

4. Reddy MA, Zhang E, Natarajan R. Epigenetic mechanisms in diabetic complications and metabolic memory. Diabetologia 2015;58:443-455 5. van Duinkerken E, Snoek FJ, de Wit M. The cognitive and psychological effects of living with type 1 diabetes: a narrative review. Diabet Med 2020;37:555-563

6. Verschueren $M$, Oris $L$, Claes $L$, Moons $P$, Weets I, Luyckx K. Identity formation in adolescents and emerging adults with type 1 diabetes. Psychol Health Med 2020;25:519-529

7. Lind M, Polonsky W, Hirsch IB, et al. Continuous glucose monitoring vs conventional therapy for glycemic control in adults with type 1 diabetes treated with multiple daily insulin injections: the GOLD randomized clinical trial. JAMA 2017;317:379-387 8. Juvenile Diabetes Research Foundation Continuous Glucose Monitoring Study Group. Effectiveness of continuous glucose monitoring in a clinical care environment: evidence from the Juvenile Diabetes Research Foundation continuous glucose monitoring (JDRF-CGM) trial. Diabetes Care 2010;33:17-22

9. DeSalvo DJ, Miller KM, Hermann JM, et al.; T1D Exchange and DPV Registries. Continuous glucose monitoring and glycemic control among youth with type 1 diabetes: international comparison from the T1D Exchange and DPV Initiative. Pediatr Diabetes 2018;19:1271-1275 10. Wadwa RP, Laffel LM, Shah VN, Garg SK. Accuracy of a factory-calibrated, real-time continuous glucose monitoring system during 10 days of use in youth and adults with diabetes. Diabetes Technol Ther 2018;20:395-402

11. National Health Service. National Diabetes Transition Audit 2003-2014, 2017. Accessed 29 November 2017. Available from https://www .digital.nhs.uk/catalogue/PUB30008

12. Abdoli S, Hardy LR, Hall J. The complexities of "struggling to live life". Diabetes Educ 2017;43: 206-215

13. Holt RIG. The burden of diabetes selfmanagement in children and young adults. Diabet Med 2017;34:747

14. Beck RW, Riddlesworth T, Ruedy K, et al.; DIAMOND Study Group. Effect of continuous glucose monitoring on glycemic control in adults with type 1 diabetes using insulin injections: the DIAMOND randomized clinical trial. JAMA 2017;317:371-378

15. Beck RW, Riddlesworth TD, Ruedy KJ, et al.; DIAMOND Study Group. Effect of initiating use of an insulin pump in adults with type 1 diabetes using multiple daily insulin injections and continuous glucose monitoring (DIAMOND): a multicentre, randomised controlled trial. Lancet Diabetes Endocrinol 2017;5:700-708

16. Vesco AT, Jedraszko AM, Garza KP, WeissbergBenchell J. Continuous glucose monitoring associ- ated with less diabetes-specific emotional distress and lower A1c among adolescents with type 1 diabetes. J Diabetes Sci Technol 2018;12:792799

17. Langendam M, Luijf YM, Hooft L, Devries JH, Mudde AH, Scholten RJ. Continuous glucose monitoring systems for type 1 diabetes mellitus. Cochrane Database Syst Rev 2012;1: CD008101

18. U.S. Food and Drug Administration. FDA News Release: FDA authorizes first fully interoperable continuous glucose monitoring system, streamlines review pathway for similar devices, 2018. Accessed 9 July 2020. Available from https:// www.fda.gov/news-events/press-announcements/ fda-authorizes-first-fully-interoperable-continuousglucose-monitoring-system-streamlines-review

19. Giani E, Snelgrove R, Volkening LK, Laffel LM. Continuous glucose monitoring (CGM) adherence in youth with type 1 diabetes: associations with biomedical and psychosocial variables. J Diabetes Sci Technol 2017;11:476-483

20. Chase HP, Beck RW, Xing D, et al. Continuous glucose monitoring in youth with type 1 diabetes: 12-month follow-up of the Juvenile Diabetes Research Foundation continuous glucose monitoring randomized trial. Diabetes Technol Ther 2010;12:507-515

21. Valenzuela JM, Records SE, Mueller KA, Martin MT, Wolf RM. Racial ethnic disparities in youth with type 1 diabetes participating in diabetes summer camps. Diabetes Care 2020;43: 903-905 


\section{AUTHOR QUERIES}

\section{PLEASE ANSWER ALL QUERIES}

Q1: Au: Based on the information on Clinicaltrials.gov, the study name is "MILLENNIALS" (for RealtiMe ContInuous gLucosE moNitoriNg With Self-monitorIng of Blood Glucose in Young AduLts With Type 1 diabeteS), should the title be updated accordingly? Also, the title is the only place in the text that the study is formally named. Should the study name be provided at its first description as a randomized crossover trial in the abstract or text, or was the study not formally named within the text intentionally?

Q2: Au: Short running title exceeded 47 characters including spaces and has been shortened to "RT-CGM Compared With SMBG in Type 1 Diabetes". If making changes, please observe character limit.

Q3: Au: Please confirm that the corresponding author's e-mail address is correct as shown.

Q4: Au: Correct expansion for MDI (multiple daily injections) inserted here at first mention?

Q5: Au: Correct expansion for GMS (Glucose Monitoring Satisfaction) inserted here and above at only two mentions in text?

Q6: Au: Correct expansion for DDS (Diabetes Distress Scale) inserted here at only mention?

Q7: Au: Word(s) missing in sentence beginning "However, in the context..."?

Q8: Au: Please expand “GOLD” study name here at only mention in text if applicable.

Q9: Au: The statement about the role of the funding source/sponsors ("Dexcom, Inc. read the manuscript. .."was duplicated here in addition to appearing in the text so that the role of the funding source is clear.

Q10: Au: Please confirm that "BI" in the Duality of Interest statement for author S.A. is correctly expanded as "Boehringer Ingelheim".

Q11: Au: Please provide the most recent date of access for reference 18 in the form of day/month/year. A temporary date of the date of copyediting has been inserted.

Q12: Au: Correct that data for the two rows of "Age range" in Table 1 are $N(\%)$ ?

Q13: Au: More of the data in Table 1 are $N(\%)$ and median (IQR) than mean plus-minus SD, so to streamline the row headings, could the legend to Table 1 be changed to "Data are $N(\%)$ or median (IQR) unless otherwise indicated" and only the rows of mean plus-minus SD data noted within Table 1 [i.e., "Age (years)", "BMI", and "HbA1c at screening, \% (mmol/mol)"]?

Q14: Au: A corresponding footnote for the dagger symbol $(\dagger)$ was not given for Table 2. Please provide the corresponding footnote or delete the symbol from the Table.

Q15: Au: A corresponding footnote for the double dagger symbol ( $\ddagger)$ was not given for Table 3. Please provide the corresponding footnote or delete the symbol from the Table. 


\section{AUTHOR QUERIES}

\section{PLEASE ANSWER ALL QUERIES}

Q16: Check that the conflict of interest information for each author is presented in full in the Duality of Interest section. 